\title{
The Probiotics in Pregnancy Study (PiP Study): rationale and design of a double- blind randomised controlled trial to improve maternal health during pregnancy and prevent infant eczema and allergy
}

Christine Barthow ${ }^{1 *}$, Kristin Wickens ${ }^{1}$, Thorsten Stanley ${ }^{2}$, Edwin A. Mitchell ${ }^{3}$, Robyn Maude ${ }^{4}$, Peter Abels ${ }^{5}$, Gordon Purdie ${ }^{6}$, Rinki Murphy ${ }^{7}$, Peter Stone ${ }^{8}$, Janice Kang ${ }^{1}$, Fiona Hood ${ }^{1}$, Judy Rowden ${ }^{3}$, Phillipa Barnes ${ }^{1}$, Penny Fitzharris ${ }^{9}$, Jeffrey Craig ${ }^{10}$, Rebecca F. Slykerman ${ }^{3}$ and Julian Crane ${ }^{1}$

\begin{abstract}
Background: Worldwide there is increasing interest in the manipulation of human gut microbiota by the use of probiotic supplements to modify or prevent a range of communicable and non-communicable diseases. Probiotic interventions administered during pregnancy and breastfeeding offer a unique opportunity to influence a range of important maternal and infant outcomes.

The aim of the Probiotics in Pregnancy Study (PiP Study) is to assess if supplementation by the probiotic Lactobacillus rhamnosus HNOO1 administered to women from early pregnancy and while breastfeeding can reduce the rates of infant eczema and atopic sensitisation at 1 year, and maternal gestational diabetes mellitus, bacterial vaginosis and Group B Streptococcal vaginal colonisation before birth, and depression and anxiety postpartum.

Methods/design: The PiP Study is a two-centre, randomised, double-blind placebo-controlled trial in Wellington and Auckland, New Zealand. Four hundred pregnant women expecting infants at high risk of allergic disease will be enrolled in the study at 14-16 weeks gestation and randomised to receive either Lactobacillus rhamnosus HN001 $\left(6 \times 10^{9}\right.$ colony-forming units per day (cfu/day)) or placebo until delivery and then continuing until 6 months post-partum, if breastfeeding.

Primary infant outcomes are the development and severity of eczema and atopic sensitisation in the first year of life. Secondary outcomes are diagnosis of maternal gestational diabetes mellitus, presence of bacterial vaginosis and vaginal carriage of Group B Streptococcus (at 35-37 weeks gestation). Other outcome measures include maternal weight gain, maternal postpartum depression and anxiety, infant birth weight, preterm birth, and rate of caesarean sections. A range of samples including maternal and infant faecal samples, maternal blood samples, cord blood and infant cord tissue samples, breast milk, infant skin swabs and infant buccal swabs will be collected for the investigation of the mechanisms of probiotic action.

(Continued on next page)
\end{abstract}

\footnotetext{
* Correspondence: christine.barthow@otago.ac.nz

${ }^{1}$ Department of Medicine, University of Otago Wellington, PO Box 7343,

Wellington South 6242, New Zealand

Full list of author information is available at the end of the article
} 
(Continued from previous page)

Discussion: The study will investigate if mother-only supplementation with Lactobacillus rhamnosus HN001 in pregnancy and while breastfeeding can reduce rates of eczema and atopic sensitisation in infants by 1 year, and reduce maternal rates of gestational diabetes mellitus, bacterial vaginosis, vaginal carriage of Group B Streptococcus before birth and maternal depression and anxiety postpartum.

Trial registration: Australian New Zealand Clinical Trials Registration: ACTRN12612000196842.

Date Registered: 15/02/12.

Keywords: Atopy, Infant eczema, Gestational diabetes, Postpartum depression, Pregnancy, Probiotic, Vaginal infections

\section{Background}

There is growing interest in the role the human microbiome has on the development of a range of chronic noncommunicable autoimmune and inflammatory conditions [1]. It is now recognised that human gut microbiota can modulate the immune system with consequences expressed both inside and outside of the gut, including prevention or modification of risk for a range of allergic, autoimmune, cardiovascular and metabolic diseases $[1,2]$. In addition, commensal gut microbiota have been demonstrated to modify host susceptibility to a range of infections [3]. Supplementation by probiotics is one approach used to intentionally modify the gut microbiota and thus influence health risks.

\section{Allergic diseases and probiotics}

Worldwide, allergic diseases are the largest group of non-communicable diseases (NCD) with an increasing prevalence in both the developed and developing world [4]. They are also the NCD with earliest onset, children bearing much of the burden of these diseases [2]. The prevalence of atopic eczema has increased two to three fold in the last three decades with 15-30\% of children worldwide [4], and up to $40 \%$ of infants in New Zealand having eczema by 15 months of age [5]. Sixty percent of children developing eczema will do so within the first year of life [4]. About half of the children who develop eczema early in life become sensitised to allergens by 2 years of age [4].

A significant body of research examining the use of probiotics to prevent allergic disease already exists. A meta-analyses of randomised controlled trials (RCT) shows benefits of using probiotic supplements during pregnancy and early infant life to prevent the development of atopic dermatitis [6]; however, a Cochrane review found that the benefit is not significant for immunoglobulin $\mathrm{E}$ (IgE) associated atopic dermatitis [7].

A more recent subgroup meta-analysis concluded that pre and post-natal supplementation is effective $(\mathrm{OR}=$ $0.61,95 \%$ confidence interval (CI) $0.52-0.71, p<0.001$ ) while there was no evidence for post-natal interventionsonly being effective $(\mathrm{OR}=0.95,95 \%$ CI $0.63-1.45, p=$
0.82) [8]. Meta-analyses also indicate both treatments with Lactobacillus alone or Lactobacillus with Bifidobacterium appear to be protective (OR $=0.70,95 \%$ CI 0.54-0.89, $p=0.004 ; \mathrm{OR}=0.62,95 \%$ CI $0.52-0.074, p<0.001$ ) [8]. This is consistent with our own previous work in a RCT of 474 infants which showed that Lactobacillus rhamnosus HNO01 (HN001) $6 \times 10^{9}$ cfu/day given daily to mothers from 35 weeks gestation, continuing until 6 months post-partum if breastfeeding and from birth until 2 years in the infant was associated with a significant $50 \%$ reduction in the prevalence of eczema at age 2 [9], 4 [10] and 6 years [11]. While not evident early, by 6 years there was also a significant reduction in skin prick sensitisation in the HN001 group $(H R=0.69$, 95 \% CI 0.48-0.99) [11].

Almost without exception [12] previous probiotic trials of allergic disease with a pre-birth intervention have commenced at some time in the final 2 months of pregnancy [6]. In our current trial we commence the intervention from 14 to 16 weeks gestation and continue it throughout pregnancy and for 6 months post-partum while breastfeeding, the probiotic being only given to the mother, not directly to the infant. Our justification for an early probiotic intervention is based on evidence showing that fetal production of IgE antibodies occurs before the end of the first trimester and allergen-specific IgE antibodies towards the end of the second trimester [13]. There is also evidence that maternal allergy alters the regulation of antigen-specific responses during pregnancy, with non-allergic mothers showing down-regulation of their (already lower) Th2 responses to specific allergen from mid to late gestation [14]. This down-regulation was absent in allergic mothers. Epidemiological support for the importance of intervention in early pregnancy comes from a longitudinal study which shows that maternal exposure to pollen during the first trimester of pregnancy increased the risk of food sensitisation in the child [15]. The majority of probiotic trials, using a late pregnancy intervention (from 32 to 35 weeks gestation), may therefore have missed the critical window to influence fetal immune responses and thus the later development of allergic disease. This may explain the general lack of effect of probiotics on 
infant sensitisation. The only study, by Huurre et al. [12], that did show a protective effect of probiotics on sensitisation in the infant used an early pregnancy intervention and the effect was limited to those with sensitised mothers $(\mathrm{OR}=0.34,95 \% \mathrm{CI} 0.13-0.88)$. In that study [12], the group of infants with non-sensitised mothers had a significantly increased risk of sensitisation but this finding was not reported in the article.

A RCT with a late pregnancy intervention [16] showed a reduction in child sensitisation also among children with allergic mothers (defined according to the presence of disease not atopic sensitisation). There was no effect among children of allergic fathers, highlighting the relative importance of the mother in influencing fetal immune development. In contrast, two probiotic trials $[17,18]$ have shown increased rates of sensitisation in all children taking probiotics but neither of these studies used Lactobacillus rhamnosus, and one intervened in infants only [18]. Confirmation of the role of probiotics in the development of atopic sensitisation in a larger study with an early pregnancy intervention may allow the targeting of a probiotic intervention to those who are most likely to benefit, i.e. in those with maternal sensitisation, while avoiding the possible increased risk of sensitisation among those without maternal sensitisation. As sensitisation is associated with more severe and persistent eczema [19], a probiotic intervention from early pregnancy, if found to protect against sensitisation, may also reduce the prevalence of clinically important eczema.

Previous pre and/or post-natal intervention studies also vary according to who received the probiotic intervention after birth: mother or infant or both. There have been two studies with an intervention only in the mothers (both from 36 weeks gestation and during breastfeeding) which have both shown an effect on eczema at 2 years that is as strong as that seen when the probiotics were also administered directly to the infant $[20,21]$. Alteration in breast milk cytokine levels associated with allergic outcomes in those receiving probiotics suggest that immune modulation may also occur through this pathway [12, 21-23], and this indicates that post-natal maternal supplementation while breastfeeding may also be important. In contrast to many of the previous probiotic studies our current study administers the probiotics directly to women only, and if proven effective provides an intervention that is easier to administer as it does not require administration of probiotics to new-born infants. This would make the intervention more easily adopted into practice.

\section{Gestational diabetes and probiotics}

Accompanying the worldwide trends in obesity, the rate of gestational diabetes mellitus (GDM) is also increasing in both the developed and the developing world [24].
Using the International Association of Diabetes and Pregnancy Study Group (IADPSG) [25] diagnostic criteria (fasting plasma glucose $\geq 5.1 \mathrm{mmol} / \mathrm{l}$, or 1 -hour post-75 g load $\geq 10.0 \mathrm{mmol} / \mathrm{l}$, or 2-hour post $75 \mathrm{~g}$ load $\geq 8.5 \mathrm{mmol} / \mathrm{l}$ ), $18 \%$ of pregnant women in the United States develop GDM during pregnancy [24]. GDM is associated with short and long-term adverse outcomes for both women and infants, including maternal gestational hypertension, polyhydramnios, preeclampsia, delivery of large-for-gestation infants, instrumental or caesarean delivery, and maternal death $[24,26]$. Adverse infant outcomes include preterm birth, shoulder dystocia, macrosomia, congenital defects, and neonatal complications such as hypoglycaemia, jaundice and respiratory distress [24]. In addition, in the longer term, women with GDM are at increased risk of metabolic syndrome [27], type 2 diabetes, and cardiovascular disease. Offspring of women with GDM have an increased risk of diabetes, obesity and metabolic issues with evidence of altered insulin secretion and lipid profiles regardless of the infant's weight [28].

Lifestyle interventions to prevent GDM relating to diet, weight loss and exercise are often unsuccessful [24, 29]; therefore primary prevention of GDM could provide substantial multigenerational health and economic benefits. In a Finnish study [30, 31], among those receiving intensive dietary counselling, probiotic use (Lactobacillus rhamnosus GG and Bifidobacterium lactis Bb12 $10^{10}$ cfu/day each) from the first trimester of pregnancy until the end of exclusive breastfeeding was associated with beneficial outcomes for GDM. The diagnostic test used in the Finnish study was a $75 \mathrm{~g}$ glucose OGTT with one value exceeding any of the following cut points being considered positive: fasting glucose value $\geq 4.8 \mathrm{mmol} / \mathrm{l}$, or $1 \mathrm{~h}$ blood glucose $\geq 10.0 \mathrm{mmol} / \mathrm{l}$, or $2 \mathrm{~h}$ blood glucose $\geq 8.7 \mathrm{mmol} / \mathrm{l}$. Using these criteria, the prevalence of GDM was dramatically decreased, $13 \%$ in women given dietary advice plus probiotics compared with $36 \%$ in a group given dietary advice only and $34 \%$ in a control group with no intervention $(p=0.003)$. The authors suggest that this effect may be due to probiotics contributing to glucose regulation during pregnancy [30]. In this same study population, probiotics taken from the first trimester were associated with half the risk of maternal adiposity, defined as having a waist circumference $\geq 80 \mathrm{~cm}$, at 6 months post-partum $(p=0.03)$ [32]. A different study using Lactobacillus rhamnosus GG supplementation from 36 weeks gestation to 6 months postnatally to breastfeeding mothers or their infant found no significant change in birth weight adjusted mean BMI in the offspring at 4 and 10 years of age [33]. A more recent study [34] using a short probiotic intervention from 24 to 28 weeks gestation and different probiotic species (Lactobacillus salivarius UCC188 $10^{9} \mathrm{cfu}$ /day) in obese pregnant woman did not alter fasting glucose or other maternal outcomes. These findings may indicate that the probiotic species and strain as well as 
gestation at commencement of intervention, duration of intervention and concurrent diet contribute to the prevention of GDM. Our current study will examine the impact of HN001 supplementation from early pregnancy without altering baseline diet.

\section{Vaginal dysbiosis and probiotics}

The maintenance of healthy vaginal microbiota is important for optimal pregnancy outcomes. Vaginal coliform and streptococcal colonisation occurs by intestinal microbes ascending from the perineum, and a healthy vaginal flora contains a predominance of organisms from the Lactobacillus genus [35]. Lactobacilli protect the vagina from pathogenic organisms by producing antimicrobial agents such as hydrogen peroxide and bacteriocins, competing for nutrients, adhering to the epithelial surfaces, maintaining the vaginal $\mathrm{pH}$ through lactic acid production, and by immune modulation [36, 37]. Both bacterial vaginosis (BV) and Group B Streptococcus (GBS) colonisation are associated with depleted vaginal lactobacillus populations [38, 39] and are associated with negative pregnancy outcomes.

Internationally the prevalence of BV is high e.g. $25 \%$ in pregnant women in USA [36]. BV is associated with preterm labour, premature rupture of membranes, spontaneous abortion, and chorioamnionitis [39]. Premature birth predisposes the infant to a range of other serious health issues including respiratory distress syndrome, intraventricular haemorrhage, leukomalacia, retinopathy, necrotising enterocolitis and prolonged hospitalisation with the associated costs to the health system [40]. Eighty percent of preterm deliveries result from premature rupture of the membranes and spontaneous preterm labour [40]. Maternal infections are associated with $30-50 \%$ of preterm labours [40].

Antibiotic therapy (metronidazole) is recommended as treatment for BV, yet a large placebo-controlled trial did not find that metronidazole reduced the occurrence of preterm delivery or other adverse perinatal outcomes [41]. However a BV treatment study showed that a combination of orally administered Lactobacillus rhamnosus GR-1 and Lactobacillus reuteri $R C-14$ and metronidazole doubled the cure rate compared to metronidazole alone [42]. Efficacy of current antibiotic treatments for BV is variable and recurrence is common (40\% at 3 months) [37]. In addition, antibiotic resistance in vaginal pathogens is an increasing concern [37]. Exploration of the role of probiotics in the prevention of BV-related adverse outcomes in pregnancy is in its infancy. A Cochrane review of probiotics for preventing preterm labour found an $81 \%$ reduction in the risk of genital infection with the use of probiotics (RR 0.19; $95 \%$ CI 0.08 to 0.48); however, there were insufficient trials to determine the effect on preterm birth and other complications [40]. Another review [43] of probiotics in the treatment and prevention of BV suggests a role for a range of Lactobacillus species in managing urogenital infections but studies with outcomes among pregnant women were absent.

GBS is a commensal bacterium found in the gastrointestinal and genitourinary tracts of $30 \%$ of healthy adults [44]. Worldwide GBS vaginal colonisation in pregnant women varies with rates of between 4 and $36 \%$ in European countries, and most countries having rates higher than $20 \%$ [44-46]. Usually maternal GBS is asymptomatic, however it can cause endometritis, chorioamnionitis, and bacteraemia in pregnant women, and may cause stillbirth [47], and is the leading cause of early onset Group B Streptococcal septicaemia and meningitis in infants [44, 48]. Up to $50 \%$ of babies born to colonised women acquire the infection and 1-2 \% of colonised infants become seriously ill [49]. Despite the low rates of early onset infant GBS illness (1-4 cases/1000 live births) the consequences are potentially fatal, including sepsis, bacteraemia, pneumonia and meningitis with associated long term neurodevelopmental defects [44, 49]. Most countries use screening to detect vaginal GBS colonisation in pregnant women at 35-37 weeks. Those colonised receive intra-partum antibiotics to reduce the risk of vertical transmission to the infant during birth.

Lactobacilli have been shown to have inhibitory effects on GBS growth in vitro $[50,51]$ and vaginal Lactobacillus counts from pregnant women are inversely related to GBS colonisation [38]. Although the popular literature supports the use of probiotics in the prevention of GBS, there has been only one feasibility study examining an oral probiotic supplementation effect on GBS, and while non-blinded and not fully powered this study did find reduced GBS colony counts in the participants taking oral probiotic supplements [35].

Studies [43], including our own [9], have shown lactobacilli survive passage through the gastrointestinal tract, indicating that oral delivery of lactobacilli is feasible and may be expected to impact the composition of vaginal flora. In addition, HNO01 has been demonstrated to produce bacteriocins [52], and appears not to have genes commonly associated with resistance to peroxide [GenBank Acc No. NZ_ABWJ00000000] so we anticipate that oral administration of this organism may favourably influence vaginal flora.

\section{Maternal postpartum depression and anxiety and probiotics}

There is a growing literature on how gut microbiota might influence anxiety, depression and cognition via the microbiota-gut-brain axis [53-56]. Much of this work has been done in preclinical animal trials by intentional manipulation of the animal's gut microbiota (such as using germ free mice, or treating with probiotics, antibiotics or pathogenic bacteria). Reviews of these 
studies demonstrate that alterations in anxiety-like or depressive behaviours in animals have been documented in response to manipulation of their gut microbiota $[54,56]$. In particular, one study has shown that probiotic supplementation with Lactobacillus rhamnosus decreased anxiety-like and depressive-like behaviours in healthy mice [57].

A range of potential mechanisms by which the gut microbiota affects central nervous system function have been proposed. These include altered microbial composition, immune inactivation, vagal nerve activation, tryptophan metabolism, gut hormone response, and through production of neuro active substances or other metabolites $[53,56]$.

There is very limited published work describing mood or cognitive outcomes of probiotic interventions in humans. In a double blind, placebo randomised controlled trial; healthy subjects were given probiotics (Lactobacillus helveticus R0052 and Bifidobacterium longum R0175) or placebo for 30 days. The probiotic group had significantly less anxiety and depression than the controls [58]. In a similar study those subjects who initially scored in the lowest third for depressed mood showed significant improvement in symptoms after probiotic treatment [59].

A recent study provides the first direct evidence that probiotics alters brain activity in humans [60]. Daily intake of a mixture of four probiotic strains (Bifidobacterium animalas subsp Lactis, Streptococcus thermophiles, Lactobacillus bulgaricus and Lactococcus lactis subsp Lactis) over four weeks reduced brain activity to an emotional attention task in the regions of the brain that influence the processing of sensory information and emotion. Brain activity was assessed using functional magnetic resonance imaging. No changes in commensal bacteria were observed. The authors argued that the probiotics might interact with the host microbiota to alter their metabolic activity resulting in the production of metabolites that influence brain activity.

The prevalence of postpartum depression is variously estimated to be 10-15\% [61], and women with postpartum depression experience a range of symptoms including general anxiety and dissatisfaction with life, emotional lability, insomnia, confusion, guilt, and suicidality [62]. In addition maternal depression can interfere with motherinfant interactions and adversely affect the infant's psychological and developmental trajectory at a time of vulnerability [62-64]. There have been no human studies examining whether probiotics given during pregnancy and breast feeding influence mood. With data emerging to indicate that probiotic effects may be mediated by the gut-brain axis, this study provides an excellent opportunity to assess this further by assessing postpartum depression and anxiety in our study population.

In summary the Probiotics in Pregnancy study aims to investigate if maternal supplementation by HN001 prevents infant eczema and atopic sensitisation by one year and improves maternal health during pregnancy by reducing GDM, BV and GBS, and in the early post-natal period by improving mood. The study design has an early maternal intervention at 14-16 weeks gestation continuing postnatally until 6 months after birth, if breastfeeding, which provides the unique opportunity to study these outcomes, all of which merit consideration on their own.

\section{Methods/design}

\section{Study design and outcomes}

The PiP Study is a two-centre randomised double blind placebo controlled trial in Wellington and Auckland, New Zealand designed to test the hypothesis that probiotic supplementation by Lactobacillus rhamnosus HN001 $\left(6 \times 10^{9} \mathrm{cfu} /\right.$ day $)$ administered to mothers from 14 to 16 weeks gestation until delivery and continuing until 6 months post-partum, if breastfeeding, will reduce (1) the prevalence of infant eczema and atopic sensitisation by age 12 months and (2) the prevalence of maternal GDM, BV and GBS vaginal colonisation before birth. Table 1 provides an overview of primary, secondary and related outcomes being examined in the study.

\section{Sample size and power calculations Eczema}

From our previous study the hazard ratio for eczema, for HN001, based on the data at 6 and 12 months, was 0.39 , with $12.3 \%$ of infants having eczema at either 6 or 12 months. With a sample size of 200 in each group and $13 \%$ drop-out rate, 43 babies are expected to be assessed as having eczema. Using the sample size formula from Therneau and Grambsch [65], the study will have $87 \%$ power at the $5 \%$ level of significance.

\section{SCORing Atopic Dermatitis (SCORAD)}

Our previous study [9] found a reduction in SCORAD [66] $\geq 10$ from 22.9 to $12.8 \%$ due to the probiotic intervention. With a sample size of 200 in each group and a $13 \%$ drop-out rate, the study will have $69 \%$ power at the $5 \%$ level of significance.

\section{Atopic sensitisation}

In general, effects of probiotics on sensitisation have only been seen in a subset of children with allergic [16] or sensitised mothers [12]. Assuming $60 \%$ of mothers are skin prick test positive and a reduction in the prevalence of atopy from 50 to $26 \%$ among their children, as seen in the paper by Huurre et al. [12], with a sample size of 200 pregnant women (120 with mothers who are sensitised) in each group and $13 \%$ drop-out rate our study will have $95 \%$ power at the $5 \%$ level of significance, for atopic sensitisation among the children of mothers that are skin prick test positive. A further analysis of the data from the study by Huurre et al. [12] shows that 
Table 1 Primary, secondary and related outcomes in the PiP Study

Primary outcomes
1. Infant eczema
2. Infant eczema severity (SCORAD $)$
3. Infant atopic sensitisation

Secondary Outcomes

1. Maternal gestational diabetes mellitus

2. Maternal BV

3. Maternal vaginal GBS colonisation

4. Maternal postpartum depression and anxiety

Adverse events and safety

1. Maternal:

Anthropometric measures

Bacteraemia/Septicaemia

Hospitalisations (excluding birth

admissions)

Gastrointestinal symptoms

Caesarean section rates
Related outcomes

Immune and inflammatory markers, epigenetic and metabolomic analyses from cord blood

Epigenetic analyses on cord tissue

Genotype and epigenetic analyses on buccal samples

Immune markers and oligosaccharide profiles from breast milk

Skin microbiota analysis from neck and axilla skin swabs and gut microbiota analysis from infant faeces

Immune and inflammatory markers, epigenetic and metabolomic analyses from cord blood

Epigenetic analyses on cord tissue

Genotype and epigenetic analyses on buccal samples

Immune markers and oligosaccharide profiles from breast milk

Skin microbiota analysis from neck and axilla skin swabs and gut microbiota analysis from infant faeces

Related Outcomes

Maternal:

Gestation at delivery.

Maternal anthropometric measures ${ }^{\mathrm{b}}$ at birth, and 6 and 12 months post-partum

Caesarean section

Fasting lipids and bile acids from fasting maternal blood taken at time of OGTT

Gut microbiota and faecal SCFA from maternal faecal samples taken in second trimester Infant:

Anthropometric measures at birth, and 6 and 12 months

Neonatal intensive care (NICU) admission

Premature rupture of membranes

Preterm birth ( $<37$ weeks)

Infant GBS

Medication use for emotional/psychological problems

2. Infant:

Anthropometric measures

Bacteraemia/Septicaemia

Hospitalisations (excluding birth admissions)

5 min Apgar scores

Preterm birth

Neonatal intensive care unit admissions

Infant symptoms

a SCORAD SCORing atopic dermatitis

${ }^{\mathrm{b}}$ Maternal height and head circumference recorded at $14-16$ weeks gestation only

there is a four-fold increased risk of sensitisation in the sub-group without maternal sensitisation. Assuming a similar difference in risk dependent on mother's atopic status, the study would have $>99 \%$ power to show an interaction effect.

\section{Gestational diabetes mellitus}

Assuming a GDM prevalence rate of $15 \%$, and based on the study by Luoto [31] which showed a $63 \%$ reduction in prevalence of diabetes mellitus in pregnant women due to a probiotic intervention, with a sample size of 200 in each group and a $2.5 \%$ drop-out rate the study will have $87 \%$ power to detect a difference at the $5 \%$ level of significance.

Bacterial vaginosis

We cannot locate any published data on the prevalence of BV in New Zealand women but international data 
suggest it is around $30 \%$ [67]. A probiotic treatment RCT of BV by Anukam [42] found a $88 \%$ cure rate (defined as normal nugent score and other markers of BV at 30 days post treatment) in the probiotic group and $40 \%$ in the placebo group. Assuming a $30 \%$ rate in the placebo group, and a similar probiotic effect resulting in a $6 \%$ rate of BV in the probiotic group, the study will have $>95 \%$ power at the $5 \%$ level of significance with a sample size of 200 in each group and $2.5 \%$ drop-out rate.

\section{Group B streptococcus}

We are not aware of any studies of a probiotic treatment effect on GBS carriage on which to base power calculations but the New Zealand prevalence was reported to be $22 \%$ in 1998-9 [46]. With a sample size of 200 in each group and a $2.5 \%$ drop-out rate, the study will have $84 \%$ power to detect a reduction to $11 \%$ at a $5 \%$ level of significance.

\section{Maternal postpartum depression and anxiety}

The distribution of the Edinburgh Postnatal Depression Scale (EPDS) from Growing up in New Zealand Study [68] was used. With a sample size of 200 in each group and $13 \%$ drop-out rate, using a Wilcoxon Rank-Sum test, the study will have $79 \%$ power to detect a $26 \%$ reduction in EPDS at a $5 \%$ level of significance.

\section{Ethics}

The study received ethical approval from the New Zealand Multi-region Ethics Committee (MEC/11/09/077). Prior to commencing the study, adult participants receive all the necessary information in relation to the study, and written informed consent is obtained. Women provide written informed consent on behalf of both themselves and their unborn child/infant.

\section{Study population \\ Pregnant women}

English-speaking pregnant women who intend to breastfeed their infant are eligible to be enrolled in the study between 14 and 16 weeks gestation if either they or the unborn child's biological father have a history of asthma or eczema treated by a doctor, or allergic rhinitis treated by a doctor or pharmacist. A woman is excluded if she: is under age 16, does not intend to stay in either of the study centers for the 18 months following enrolment, has a serious immunological disorder that suppresses immune function or is taking immune suppressant drugs, has known cardiac valve disease for which antibiotic prophylaxis is required when undergoing dental procedures, has a history of a transplant or human immunodeficiency virus, were on long-term of continuous antibiotic therapy, required in vitro fertilisation to establish the current pregnancy, has a pre-enrolment scan showing major fetal abnormalities, at the time of enrolment is using or intended to use probiotic drinks or supplements themselves or in their child, is participating in another randomised controlled trial, has a severe allergy to cow's milk (as probiotic capsules may contain traces of milk), has previously participated in the study with an older child or is deemed unsuitable for study inclusion for any other medical reason. Any pregnant woman with pre-existing type 1 or 2 diabetes is eligible for the study but is excluded from the oral gestational tolerance test (OGTT) and gestational diabetes outcomes.

\section{Infants}

All infants born in the study are eligible for inclusion in study outcomes. In the case of multiple births, only the first-born infant will be included in the study.

\section{Fathers}

The biological father of the infant is invited to participate in a study visit to obtain some background data relating to their history of allergic disease, atopic sensitisation by skin testing and anthropometric measures. These data are being collected to enable examination of the relationship between paternal characteristics and infant growth, allergic disease and atopic sensitisation.

\section{Recruitment}

A range of recruitment strategies are used to notify pregnant women about the study and to invite them to contact the study centres to discuss the study and be assessed for study eligibility. These include: enclosing study brochures in information packs routinely given to women by clinicians and midwives in early pregnancy, emailing employees of large organisations, using webbased approaches such as Facebook, arranging for sonographers and phlebotomists to hand out study brochures at the time of early scans and the first antenatal blood collection, radio advertising, newspaper articles as well as pamphlets and posters placed at hospitals, clinics, crèches, libraries and pharmacies and inviting clinicians (midwives, obstetricians and general practitioners) to refer women to the study.

Pregnant women who contact the study centres are screened for eligibility by phone and if eligible are invited to attend an enrolment visit at the study centre when they reach between 14 and 16 weeks gestation.

\section{Study intervention \\ Capsules}

Study capsules containing Lactobacillus rhamnosus HN001 $\left(6 \times 10^{9} \mathrm{cfu}\right)$ are manufactured by Fonterra Cooperative Group Ltd as previously described [9]. Shelf life is managed to ensure minimum viable counts of $6 \times 10^{9} \mathrm{cfu}$ are maintained in the HN001 capsules. The 
placebo powder is corn-derived maltodextrin, manufactured by Grain Processing Corp. Oregon, USA and is supplied to Fonterra Co-operative Group Ltd by Salkat New Zealand Ltd, Auckland. Placebo capsules have identical appearance and smell to the probiotic capsules. Both probiotic and placebo powders are encapsulated by Alaron Products Ltd, Nelson, New Zealand and provided in opaque bottles. Quality and safety testing is performed to a pharmaceutical standard (Therapeutic Goods Act) by a registered external laboratory.

\section{Randomisation process}

Randomisation is managed by Fonterra Co-operative Group Ltd and concealed from all study staff and participants. Randomisation is stratified by study centre and performed in blocks of random lengths according to a computer-generated randomisation list. At enrolment, a research staff member assigns the woman the next consecutive study number and provides her with the appropriate capsules.

\section{Capsule viability and compliance}

All capsules are stored at $4^{\circ}$ Celsius $\left({ }^{\circ} \mathrm{C}\right)$ in the study centres and participants are advised to store the capsules in their home refrigerator. Written and verbal instructions regarding use and storage of capsules are provided to participants. Women are advised to take one study capsule daily from enrolment until 6 months after birth if breastfeeding, or to cease capsules before 6 month post birth if they have not breastfed for $24 \mathrm{~h}$. To assess compliance, capsule bottles are collected at regular intervals and counts of remaining capsules are completed by an independent person. Samples of capsules returned from the field are tested to ensure that capsules have maintained their viability. As previously described [9], viable bacteria are counted using standard methods, and counts checked against samples of HN001 kept at $4{ }^{\circ} \mathrm{C}$.

\section{Study assessments}

Table 2 provides a summary of the study timeline and investigations. Women enrolled in the study are provided with a study calendar to assist them to prospectively record data relevant to the study questionnaires. Research staff members are trained in standardised administration of all study questionnaires and data collection procedures.

Questionnaires collect a range of data including confounders (such as previous GDM, parity etc.), effect modifiers (such as use of antibiotics, yoghurt etc.), outcome data, and adverse events.

\section{Assessment of primary outcomes}

Infant eczema After withholding topical creams and oils for $24 \mathrm{~h}$ the prevalence of eczema in infants is assessed at 6 and 12 months using the UK Working Party's Diagnostic Criteria for atopic dermatitis [69] which is adapted for infants under 12 months [9]. All research staff members undertake online training in the application of this tool. Eczema is assessed as present at each visit if there is a history of scratching or rubbing and two or more of the following occurring since birth or the previous visit: (1) a history of involvement of outer arms or legs, (2) a history of a generally dry skin, or (3) visible eczema present on the cheeks or outer arms or legs with no axillary involvement. Eczema severity is assessed at the same time points using SCORAD [66] with staff recording any region of greater than or equal to $1 \mathrm{~cm}$ diameter that meets the skin change criteria [69] for eczema. For the purpose of consistency between the UK Working Party's definition of atopic dermatitis and SCORAD, papulation is considered a surface change when using these tools.

Atopic sensitisation Skin prick tests are performed using standardised protocols, (after relevant antihistamine withhold periods if needed) using Stallergenes $1 \mathrm{~mm}$ lancets (Antony, France), positive control (histamine $10 \mathrm{mg} / \mathrm{ml}$ ), negative control (phenolated glycerolsaline), Dermatophagoides pteronyssinus, cat fur, five grass mix (cocksfoot, sweet vernal, rye, timothy and meadow grass), (Alyostal Stallergenes, Antony, France), egg white, peanut and cow's milk (ALK- ABELLO, Madrid, Spain). Both biological parents are tested with positive, negative controls and aeroallergens only. Infants are tested at 12 months using both controls and all allergens. After application allergens are pricked vertically for $1 \mathrm{~s}$, positive control wheals are measured at $10 \mathrm{~min}$, and the negative control and all allergens are measured at $15 \mathrm{~min}$ to the nearest $\mathrm{mm}$. The wheal size is calculated as the mean of the longest diameter and the midpoint perpendicular diameter. After the subtraction of any reaction to the negative control a mean wheal diameter of $3 \mathrm{~mm}$ or greater will be considered a positive reaction.

\section{Assessment of secondary outcomes}

Gestational diabetes mellitus Women who are diagnosed with diabetes prior to 20 weeks gestation are excluded from the gestational diabetes outcomes for the study, as this diagnosis is likely to reflect pre-existing diabetes. Gestational diabetes outcomes are assessed at 26-28 weeks gestation, by a three time-point test $75 \mathrm{~g}$ oral glucose tolerance test (OGTT) recommended by international guidelines [25]. Preparation for the test include a 10-16 h pretest fast with instructions to avoid smoking during this time and during the test, and avoidance of exercise on the morning of the test. Participants remain in a resting state 
Table 2 Study timeline and investigations

\begin{tabular}{|c|c|c|c|c|c|c|c|c|}
\hline & $\begin{array}{l}\text { 14-16 Weeks } \\
\text { Gestation }\end{array}$ & $\begin{array}{l}\text { 26-28 Weeks } \\
\text { Gestation }\end{array}$ & $\begin{array}{l}\text { 35-37 Weeks } \\
\text { Gestation }\end{array}$ & Birth & $\begin{array}{l}\text { 3-7 Days } \\
\text { After Birth }\end{array}$ & $\begin{array}{l}\text { Infant Age } 3 \\
\text { Months }\end{array}$ & $\begin{array}{l}\text { Infant Age } 6 \\
\text { Months }\end{array}$ & $\begin{array}{l}\text { Infant Age } 12 \\
\text { Months }\end{array}$ \\
\hline $\begin{array}{l}\text { Intervention: new bottle } \\
\text { study capsules }\end{array}$ & $\begin{array}{l}\checkmark \text { Intervention } \\
\text { commences }\end{array}$ & $\checkmark$ & & & $\checkmark$ & $\checkmark$ & $\begin{array}{l}\text { Intervention } \\
\text { stops }\end{array}$ & \\
\hline Questionnaire & $\checkmark$ & $\checkmark$ & & & $\checkmark$ & $\checkmark$ & $\checkmark$ & $\checkmark$ \\
\hline \multicolumn{9}{|c|}{ Objective measures and samples: Maternal } \\
\hline Vaginal swab (BV) & $\checkmark$ & & $\checkmark$ & & & & & \\
\hline Vagino-rectal swab (GBS) & $\checkmark$ & & $\checkmark$ & & & & & \\
\hline Anthropometry ${ }^{\mathrm{a}, \mathrm{b}, \mathrm{c}, \mathrm{d}}$ & $\checkmark$ & & & & $\checkmark$ & & $\checkmark$ & $\checkmark$ \\
\hline $\begin{array}{l}\text { Blood samples (OGTT and } \\
\text { serum) }\end{array}$ & & $\sqrt{ }^{\mathrm{e}}$ & & & & & & \\
\hline Faecal sample & & $\checkmark$ & & & & & & \\
\hline 3 day food diary & & $\checkmark$ & & & & & & \\
\hline Cord blood and tissue & & & & $\checkmark$ & & & & \\
\hline Breast milk & & & & & $\checkmark$ & & & \\
\hline Skin prick test & & & & & & & $\sqrt{ }$ Completed & fter birth \\
\hline \multicolumn{9}{|c|}{ Objective measures and samples: Infant } \\
\hline Anthropometry ${ }^{\mathrm{a}, \mathrm{b}, \mathrm{c}}$ & & & & & $\checkmark$ & & $\checkmark$ & $\checkmark$ \\
\hline Eczema & & & & & & & $\checkmark$ & $\checkmark$ \\
\hline SCORAD & & & & & & & $\checkmark$ & $\checkmark$ \\
\hline Faecal sample & & & & & & & & $\checkmark$ \\
\hline 48 hour food diary & & & & & & & & $\checkmark$ \\
\hline Buccal sample & & & & & & & & $\checkmark$ \\
\hline Skin swabs & & & & & & & & $\checkmark$ \\
\hline Skin prick test & & & & & & & & $\checkmark$ \\
\hline \multicolumn{9}{|c|}{ Objective measures and samples: Paternal } \\
\hline Anthropometry ${ }^{a, b, c}$ & & & & & \multicolumn{4}{|c|}{$\boldsymbol{\checkmark}$ Completed at any visit after birth } \\
\hline Skin prick test & & & & & \multicolumn{4}{|c|}{$\checkmark$ Completed at any visit after birth } \\
\hline
\end{tabular}

$B V$ bacterial vaginosis, GBS group B streptococcus, OGTT oral glucose tolerance test, SCORAD scoring atopic dermatitis

a height/length ${ }^{b}$ weight ${ }^{c}$ head circumference ${ }^{d}$ waist circumference ${ }^{e}$ excluding women with pre-existing diabetes

during the test which comprises of a baseline fasting blood sample, $75 \mathrm{~g}$ glucose load and blood samples at 1 and $2 \mathrm{~h}$ post load. An additional blood sample is collected at the time of the fasting blood test and this sample is separated and the serum stored at $-80{ }^{\circ} \mathrm{C}$ for future analysis.

BV and GBS All swabs and blood samples are processed using standard laboratory practices by accredited medical laboratories in the study centres. Vaginal swabs for BV and vagino-rectal swabs for GBS are self-collected by woman at enrolment (baseline) and 35-37 weeks gestation (outcome) following standardised written instructions and using Copan Transystem Amies (Ref 108CF.FR) swabs. Additional file 1: Figure S1 shows the swab collection instructions in more detail. After collection, swabs are transported at ambient temperature to laboratories as soon as is possible but within a maximum of $24 \mathrm{~h}$. BV swabs are gram stained, and reported according to the three bands of Nugent's scale, (0-3 being normal, 4-6 intermediate, and 7 or more indicating BV) [70]. GBS swabs are reported according to the presence or absence of GBS. As GBS is not treated in early pregnancy and BV is only treated if symptomatic, the results of baseline swabs remain confidential to the study. This is to ensure study swabs do not unintentionally result in unrequired antibiotic treatment being prescribed by reporting positive swabs which may not be from symptomatic disease. Only the results of the outcome 35-37 week GBS swabs are shared with the woman's lead maternity carer $(\mathrm{LMC})^{1}$ as these swabs inform the woman's clinical care.

Maternal postpartum depression and anxiety At 12 months post birth or later women will be asked to provide retrospective information related to their mood at 1-2 months after birth. Questions include the EPDS [71], state anxiety inventory [72], with the addition of questions to collect medication use for emotional and psychological problems. 


\section{Other measures}

Anthropometric measures Anthropometric measures are being performed using regularly calibrated standardised equipment and according to protocols adapted from the World Health Organisation [73] (see Additional file 2: Table S3).

Food diaries and faecal samples Three day food diaries and faecal samples are collected from women at the time of their OGTT, and infants at 12 months. Participants are requested to prospectively record all foods and drinks taken during the diary period including the amounts taken, methods of cooking, any additions to the food (e.g. sauces) and any dietary supplements taken during the period. The food dairy is completed in the 2-3 days immediately prior to the collection of a faecal sample.

Faecal samples are collected into clean containers, then a small portion of faecal material is immediately transferred into hospital grade faecal sample pots and frozen at $-20{ }^{\circ} \mathrm{C}$ with the sample container surrounded by water to ensure the sample remains frozen while being transferred to the research centre. Samples are collected no more than $48 \mathrm{~h}$ before being transferred to the research centres and stored at $-80{ }^{\circ} \mathrm{C}$. A range of quality control data are collected regarding these samples, detailed in Additional file 3: Table S4.

Cord blood and tissue samples Cord blood and cord tissue samples are collected from participants in Wellington only. These samples are being collected for a range of studies to elucidate the mechanisms of probiotic action including cytokine, epigenetic and metabolomic studies (see Table 1). Using standardised instructions, intra-partum carers collect up to $40 \mathrm{ml}$ of cord blood using an 18 gauge needle and sterile syringe, and 2 cord tissue samples. Further details of sample collection, processing and quality control data are presented in Additional file 4: Table S5.

Breast milk samples Pre-feed breast milk samples are collected by women at 4-7 days after birth after the breast milk has come in. Women are advised to wash their hands prior to sample collection, then express a few drops of milk and discard before expressing a 10-15 ml into a sterile polypropylene $70 \mathrm{ml}$ container. This sample is divided between three $5 \mathrm{ml}$ sterile leak-proof freestanding polypropylene polyethylene Rnase and Dnase free tubes (Interlab code 3811-345-008). Samples are chilled in the home refrigerator if the sample will be picked up by research staff within $48 \mathrm{~h}$ or frozen in the home freezer with water surrounding the milk tubes if the sample will be picked up more than $48 \mathrm{~h}$ after collection [74]. All samples are stored at $-80{ }^{\circ} \mathrm{C}$. Quality data collected for these samples are documented in Additional file 5: Table S6.

Infant skin swabs To enable examination of the infant skin microbiota two skin swabs are taken, one from the infants left neck (a common site for eczema) and another from the left axilla (a site where eczema is uncommon) at the 12 month visit using protocols adapted from the manual of procedures from the Human Microbiome Project [75]. Prior to sampling, parents are advised to avoid the following: antibiotic or steroid creams for 7 days (if possible), antiseptic or antimicrobial soaps or bath additives for $48 \mathrm{~h}$, swimming for $48 \mathrm{~h}$, washing for $24 \mathrm{~h}$, and any creams or oils on the skin on the day of the visit. Questionnaire data is obtained to assess compliance with these preparations and to collect data related to any antibiotic use which may alter swab outcomes. To avoid possible contamination of samples with the researcher's skin microbiota, these samples are collected at the commencement of the study visit and prior to the researcher having other physical contact with the infant. Samples are collected using sterile gloves and aseptic technique and Eswabs containing Amies fluid (480CE ESwab LQ Amies Reg. Nylon Flocked Applicator, Copan). Neck swabs are taken from the left side of the neck midway down a vertical line from earlobe to base of neck. The swab is moistened in Amies fluid and then using firm pressure and covering an area of $4 \mathrm{~cm}^{2}$ swabbed back and forth five times (one complete swab of back and forth is counted as one cycle). The swab is then rotated so the opposite swab surface is touching child's skin and rubbed back and forth again in the same area for a further five cycles. A second swab is taken from the left axilla in the same manner. Following collection, swabs are immediately placed on ice prior to storage at $-80{ }^{\circ} \mathrm{C}$. Quality data includes date and time of swab collection and placement at $-80{ }^{\circ} \mathrm{C}$, and if full 10 cycles of swabbing is completed.

Infant buccal sample Two infant buccal samples are taken at 12 months to enable future genotyping and epigenetic analysis. To prevent sample contamination parents are advised to avoid giving their child anything other than water to eat or drink, and to avoid cleaning teeth in the hour prior to sample collection. Research staff wear clean gloves and samples are collected using sterile Copan 502CS01 regular flocked swabs and by rubbing up and down 10 times against the inside of the cheek then gently rubbing in the grooves next to the top and bottom gums on the same side for $10 \mathrm{~s}$. Swabs are placed in sterile $5 \mathrm{ml}$ sterile SIMP309-5A cryovials and immediately placed on ice until transferred to a $-80{ }^{\circ} \mathrm{C}$ freezer. Date and time of swab collection and time of placement at $-80{ }^{\circ} \mathrm{C}$ are recorded. The child's grandparents and mother's ethnicity are recorded. 


\section{Safety and adverse event monitoring}

Our previous study using HNO01 in women from 35 weeks gestation until 6 months post birth and in their infants for 2 years found no adverse effects [76]. Similarly other studies of safety using differing strains of Lactobacillus rhamnosus [77], and studies with interventions commencing from the first trimester [31, 78] have found no adverse effects on maternal (gestation length, miscarriage, caesarean delivery) or infant (Apgar score, birth weight and length, head circumference) outcomes.

Adverse event data are collected in all questionnaires. In addition, study doctors review the notes of any participant where there is any event that may require review, such as if the infant is delivered at less than 37 weeks' gestation; if an infant requires resuscitation at birth; if the infant has any malformations (apart from tongue tie); if the infant requires admission to neonatal unit for more than $48 \mathrm{~h}$ for any reason; if the woman or baby had any hospital admission other than the birth admission; if the women or infant have any serious infection (e.g. septicaemia) or any other serious health issues.

\section{Data analysis}

An intention-to-treat analysis will be used to assess the effect of HNO01 on the 12 month cumulative prevalence of eczema and SCORAD $\geq 10$ using hazard ratios from a Cox's proportional hazards model, and the point prevalence of atopy at age 12 months using relative rates and a chisquared test. Chi-square test will also be used to compare the proportion in each study group with detectable levels of immunological markers in breast milk and for those with detectable levels, t-tests will be used to compare the groups if the data is log-normally distributed. If the data is not log-normally distributed a Wilcoxon Rank-Sum test will be used. Where there are important differences between groups, these variables will be added to the models to assess whether these variables mediate any effect of HNO01 on eczema and/or atopic sensitisation (using a generalised linear model with a log link and binomial distribution). The association of HNO01 with the presence of GDM, BV and GBS will be assessed using relative rates and chisquared tests. EPDS and State Anxiety Inventory will be compared with a Wilcoxon Rank-Sum test. The effect of weight gain will be investigated as an intermediate variable by adding it to the model (a generalised linear model with a log link and binomial distribution) in the GDM analysis. Any baseline differences that occur will be adjusted for. If commercially available non-study probiotics have been used, a sub-group analysis will be performed after excluding this group, which we do not expect to be large. Reasons for withdrawal from the study will be recorded and examined for differences between study groups. Adjustment will be made for differences in antibiotic use between study groups. SAS version 9.4 will be used.

\section{Discussion}

In spite of the growing body of evidence for the benefits of probiotics in prevention of eczema these interventions have not been adopted into routine clinical practice [79]. The heterogeneity of primary prevention studies with different combinations of probiotics, strains, doses and timeframes for interventions makes meaningful synthesis of studies difficult. A recent 2015 World Allergy Organisation guideline has for the first time suggested that pregnant women whose infants are at high risk for allergy should take probiotics; however this recommendation is recorded as conditional and based on very low quality evidence [80]. In spite of the need for more research in this area, undertaking such studies is complex. Commercially available probiotic supplements are increasing in number and are readily accessible. In conjunction with this we believe that there is an increasing community acceptance and confidence in the benefits probiotic supplementation provide. Recruitment to the PiP Study is slower than originally planned partly because some eligible pregnant women decline study enrolment because they did not wish to be randomised to receive the placebo, choosing instead to purchase their own probiotic supplements.

We routinely discourage use of probiotic supplements other than the study capsules, but so we can account for any non-study probiotic exposure, we record details of any that are taken. We also collect data on use of yoghurts which are the most commonly consumed food sources of probiotics in New Zealand. We notice an increasing array of foods and drinks on the market which contain probiotics, and while many of these may be inactive or have minimal effectiveness such exposures are not easily incorporated into study data collection. These issues create significant challenges in the process of building a reliable and strong evidence base to enable confident recommendations to be made in clinical settings.

In comparison with our previous work with HN001 [9-11] showing a protective effect for the development of eczema the design of this study changes several things. This includes the different timeframe for the commencement of the intervention (14-16 weeks gestation compared to 36 weeks gestation previously), with only the women receiving the probiotic supplementation (compared to both women and infant previously), and final allergic outcomes will be measured at age 12 months (compared to 24 months previously). If this study does not demonstrate a protective effect for development of eczema it will be difficult to interpret which of these factors has caused a change in effect.

\section{Clinical significance}

The PiP Study will assess the efficacy of HN001 supplementation from 14 to 16 weeks gestation in the prevention of GDM, BV, and vaginal GBS colonisation in 
pregnant women, and development of eczema and atopic sensitisation in their infants at one year and maternal depression and anxiety at 1-2 months postpartum. A unique strength of the study is the range of maternal and infant outcomes examined as a result of a single intervention. If successful the intervention could be adopted into practice with relative ease. This is in contrast to other probiotic eczema prevention strategies which also require direct infant supplementation. Through analysis of biological samples collected prospectively the study may also provide insights into the mechanisms of probiotic action in prevention of gestational diabetes, infant eczema and infant atopic disease. In addition the study will provide further valuable information of the safety of the HNO01 supplementation in these populations.

\section{Trial status}

The PiP Study is ongoing, and final outcome data are due to be collected by mid-2016.

\section{Endnotes}

${ }^{1}$ In New Zealand, maternity care and delivery is managed through a system of lead maternity carers. These carers provide maternity care and support through the pregnancy, co-ordinate with other healthcare providers, organise scans and tests, manage the labour and provide care for the first six weeks of the baby's life. Most lead maternity carers are midwives, though some doctors and obstetricians may also carry out the role.

\section{Additional files}

Additional file 1: Figure S1. Instructions for taking vaginal and vagino-rectal swabs for the Probiotics in Pregnancy Study. Description: Detailed instructions for study participants to take vaginal and vagino-rectal swabs. (PDF $165 \mathrm{~kb}$ )

Additional file 2: Table S3. Anthropometric measures: equipment and protocols. Description: Equipment and protocols for anthropometric measures. (PDF $37 \mathrm{~kb}$ )

Additional file 3: Table S4. Quality data for faecal samples. Description: list of quality control data recorded for infant and maternal faecal samples. (PDF $30 \mathrm{~kb}$ )

Additional file 4: Table S5. Cord blood and tissue collection, processing and quality data. Description: Description of collection equipment, laboratory processing and quality control data for cord blood and tissue samples. (PDF $36 \mathrm{~kb}$ )

Additional file 5: Table S6. Quality data for breast milk samples. Description: Details of data recorded for quality control of breast milk samples. (PDF $30 \mathrm{~kb}$ )

\section{Abbreviations}

$\mathrm{BV}$, bacterial vaginosis; ${ }^{\circ} \mathrm{C}$, degrees Celsius; cfu, colony-forming units; cfu day ${ }^{-1}$, colony-forming units per day; EPDS, Edinburg postnatal depression scale; GDM, gestational diabetes mellitus; GBS, group B streptococcus; HN001, Lactobacillus rhamnosus HN001; NCD, non communicable diseases; OGTT, oral glucose tolerance test; PiP Study, Probiotics in Pregnancy Study; RCT, randomised controlled trial; SCFA, short chain fatty acids

\section{Acknowledgements}

This study is funded by grants and support from the: Health Research Council of New Zealand (HRC 11/318); Fonterra Co-operative Group Ltd, New Zealand; Nurture Foundation for Reproductive Research, New Zealand; and the University of Otago, Wellington, New Zealand. EAM and RFS are supported by Cure Kids.

The authors thank: Professor Susan Prescott (University of Western Australia) for protocols for separation of cord bloods for cytokines; Kathryn Isaac and Rachel Beddow, (Wellington Regional Genetics Laboratory, Wellington Hospital, New Zealand) for modification and testing of protocol for separation and storage of cord bloods for cytokines; Dr Elizabeth Forbes-Blom and Dr Lieke van den Elsen, (Malaghan Institute of Medical Research, Wellington, New Zealand) for advice and analysis of breast milk cytokines and IgA; Dr Deborah Williamson, (University of Otago, Wellington, New Zealand) for advice on protocols for collection of skin swabs; Sherron Kneebone, Amy Norman and Barbara Wright for assistance with Auckland study recruitment and fieldwork.

\section{Availability of data and material}

This manuscript reports study protocols only, therefore no data are reported or available.

\section{Authors' contributions}

KW conceived the study, led study design and coordination, and commented on the manuscript. CB assisted with study design and drafted the manuscript. JR coordinated the field research team in Auckland, assisted with Auckland field work and assisted with the design of maternal post-partum depression and anxiety components of the study. PB assisted with study design and in particular the development of study databases. J. Craig advised regarding development of protocols for cord blood and tissue samples collected for epigenetics, metabolomics, CRP and infant buccal samples. RFS reviewed literature related to microbiota-gut-brain axis and advised on study design relating to maternal post-partum depression and anxiety. All other authors assisted with study design and commented on the manuscript. All authors read and approved the final manuscript.

\section{Competing interests}

Fonterra Co-operative Group Ltd, New Zealand made a contribution to funding and provided the probiotic and placebo capsules.

The authors declare that they have no competing interests.

\section{Consent for publication}

Not applicable.

\section{Ethics approval and consent to participate}

Ethics approval was granted by the New Zealand Multi-region Ethics Committee (MEC/11/09/077). Written informed consent is obtained from adult participants. Women provide written informed consent on behalf of both themselves and their unborn child/infant.

\section{Author details}

'Department of Medicine, University of Otago Wellington, PO Box 7343, Wellington South 6242, New Zealand. ${ }^{2}$ Department of Paediatrics, University of Otago Wellington, PO Box 7343, Wellington South 6242, New Zealand.

${ }^{3}$ Department of Paediatrics: Child and Youth Health, University of Auckland, Private Bag 92019, Auckland 1142, New Zealand. ${ }^{4}$ Graduate School of Nursing, Midwifery and Health, Victoria University of Wellington, P O Box 7625, Newtown, Wellington 6242, New Zealand. ${ }^{5}$ Department of Obstetrics and Gynaecology, University of Otago Wellington, PO Box 7343, Wellington South 6242, New Zealand. ${ }^{6}$ Dean's Department, University of Otago Wellington, PO Box 7343, Wellington South 6242, New Zealand. 'Department of Medicine, University of Auckland, Private Bag 92019, Auckland 1142, New Zealand. ${ }^{8}$ Department of Obstetrics and Gynaecology, University of Auckland, Private Bag 92019, Auckland 1142, New Zealand. ${ }^{9}$ Immunology Department, Auckland Hospital, Private Bag 92024, Auckland Mail Centre, Auckland 1142, New Zealand. ${ }^{10}$ Murdoch Children's Research Institute, The Royal Children's Hospital, Flemington Road, Parkville, Melbourne, Victoria 3052, Australia.

Received: 28 September 2015 Accepted: 26 May 2016 Published online: 03 June 2016 


\section{References}

1. Cénit MC, Matzaraki V, Tigchelaar EF, Zhernakova A. Rapidly expanding knowledge on the role of the gut microbiome in health and disease. Biochim Biophys Acta. 2014;1842:1981-92.

2. Prescott SL. Early-life environmental determinants of allergic diseases and the wider pandemic of inflammatory noncommunicable diseases. J Allergy Clin Immunol. 2013;131:23-30.

3. Wallace TC, Guarner F, Madsen K, Cabana MD, Gibson G, Hentges E, et al. Human gut microbiota and its relationship to health and disease. Nutr Rev. 2011;69:392-403.

4. Pawankar R, Canonica RW, Holgate ST, Lockey RL, Blaiss M, editors. World Allergy Organisation (WAO) White Book on Allergy: update 2013. Milwaukee: World Allergy Organization; 2013.

5. Silvers KM, Frampton CM, Wickens K, Epton MJ, Pattemore PK, Ingham T, et al. Breastfeeding protects against adverse respiratory outcomes at 15 months of age. Matern Child Nutr. 2009;5:243-50.

6. Pelucchi C, Chatenoud L, Turati F, Galeone C, Moja L, Bach J-F, et al. Probiotics supplementation during pregnancy or infancy for the prevention of atopic dermatitis: A meta-analysis. Epidemiology. 2012;23:402-14

7. Osborn DA, Sinn JKH. Probiotics in infants for prevention of allergic disease and food hypersensitivity. Cochrane Database Syst. Rev. 2007; Art. No.: CD006475. DOl: 10.1002/14651858.CD006475.

8. Panduru M, Panduru NM, Sălăvăstru CM, Tiplica G-S. Probiotics and primary prevention of atopic dermatitis: a meta-analysis of randomized controlled studies. J Eur Acad Dermatol Venereol. 2015;29:232-42.

9. Wickens K, Black PN, Stanley TV, Mitchell E, Fitzharris P, Tannock GW, et al. A differential effect of 2 probiotics in the prevention of eczema and atopy: a double-blind, randomized, placebo-controlled trial. J Allergy Clin Immunol. 2008;122:788-94.

10. Wickens K, Black P, Stanley TV, Mitchell E, Barthow C, Fitzharris P, et al. A protective effect of Lactobacillus rhamnosus HN001 against eczema in the first 2 years of life persists to age 4 years. Clin Exp Allergy. 2012;42:1071-9.

11. Wickens K, Stanley TV, Mitchell EA, Barthow C, Fitzharris P, Purdie G, et al. Early supplementation with Lactobacillus rhamnosus HN001 reduces eczema prevalence to 6 years: Does it also reduce atopic sensitization? Clin Exp Allergy. 2013;43:1048-57.

12. Huurre A, Laitinen K, Rautava S, Korkeamäki M, Isolauri E. Impact of maternal atopy and probiotic supplementation during pregnancy on infant sensitization: a double-blind placebo-controlled study. Clin Exp Allergy. 2008;38:1342-8

13. Hertz-Picciotto I, Park H-Y, Dostal M, Kocan A, Trnovec T, Sram R. Prenatal exposures to persistent and non-persistent organic compounds and effects on immune system development. Basic Clin Pharmacol Toxicol. 2008;102:146-54.

14. Breckler LA, Hale J, Jung W, Westcott L, Dunstan JA, Thornton CA, et al. Modulation of in vivo and in vitro cytokine production over the course of pregnancy in allergic and non-allergic mothers. Pediatr Allergy Immunol. 2010;21:14-21.

15. Pyrhonen K, Laara E, Hiltunen L, Kaila M, Nayha S. Season of the first trimester in pregnancy predicts senstization to food allergens in childhood: a population-based cohort study from Finland. J Epidemiol Community Heal. 2010; doi:10.1136/jech.2009.105411.

16. Abrahamsson $T$, Jakobsson $T$, Bottcher $M$, Fredrikson $M$, Jenmalm MC, Bjorksten B, et al. Probiotics in prevention of IgE-associated eczema: A double-blind, randomized, placebo-controlled trial. J Allergy Clin Immunol. 2007;119:1174-80

17. Niers L, Martin R, Rijkers G, Sengers F, Timmerman H, van Uden N, et al. The effects of selected probiotic strains on the development of eczema (the PandA study). Allergy. 2009;64:1349-58.

18. Taylor AL, Dunstan JA, Prescott SL. Probiotic supplementation for the first 6 months of life fails to reduce the risk of atopic dermatitis and increases the risk of allergen sensitization in high-risk children: A randomized controlled trial. J Allergy Clin Immunol. 2007;119:184-91.

19. Flohr $\mathrm{C}$, Johansson $\mathrm{S}$, Wahlgren $\mathrm{C}-\mathrm{F}$, Williams $\mathrm{H}$. How atopic is atopic dermatitis? J Allergy Clin Immunol. 2004;114:150-8.

20. Dotterud CK, Storro O, Johnsen R, Oien T, Storrø O, Johnsen R, et al. Probiotics in pregnant women to prevent allergic disease: a randomized, double-blind trial. Br J Dermatol. 2010;163:616-23.

21. Rautava S, Kalliomaki M, Isolauri E. Probiotics during pregnancy and breastfeeding might confer immunological protection against atopic disease in the infant. J Allergy Clin Immunol. 2002;109:119-21.
22. Prescott SL, Wickens K, Westcott L, Jung W, Currie H, Black PN, et al. Supplementation with Lactobacillus rhamnosus or Bifidobacterium lactis probiotics in pregnancy increases cord blood interferon-y and breast milk transforming growth factor- $\beta$ and immunoglobin A detection. Clin Exp Allergy. 2008;38:1606-14.

23. Bottcher M, Abrahamsson T, Fredriksson M, Jakobsson T, Bjorksten B. Low breast milk TGF-B2 is induced by Lactobacillus reuteri supplmentation and associates with reduced risk of sensitization during infancy. Pediatr Allergy Immunol. 2008;19:497-504

24. Barrett HL, Dekker Nitert M, Conwell LS, Callaway LK. Probiotics for preventing gestational diabetes. Cochrane Database Syst Rev. 2014; Art. No. CD009951. DOl: 10.1002/14651858.CD009951.

25. International Association of Diabetes and Pregnancy Study Groups Consensus Panel. International Association of Diabetes and Pregnancy Study Groups recommendations on the diagnosis and classification of hyperglycemia in pregnancy. Diabetes Care. 2010;33:676-82.

26. Poston L, Harthoorn LF, Van Der Beek EM. Obesity in pregnancy: Implications for the mother and lifelong health of the child. A consensus statement. Pediatr Res. 2011;69:175-80.

27. Vohr BR, Boney CM. Gestational diabetes: the forerunner for the development of maternal and childhood obesity and metabolic syndrome? J Matern Neonatal Med. 2008;21:149-57.

28. Chandler-Laney PC, Bush NC, Granger WM, Rouse DJ, Mancuso MS, Gower BA. Overweight status and intrauterine exposure to gestationa diabetes are associated with children's metabolic health. Pediatr Obes. 2011;7:44-52

29. Nitert MD, Barrett HL, Foxcroft K, Tremellen A, Wilkinson S, Lingwood B, et al. SPRING: an RCT study of probiotics in the prevention of gestational diabetes mellitus in overweight and obese women. BMC Pregnancy Childbirth. 2013:13:50

30. Laitinen K, Poussa T, Isolauri E, the Nutrition Allergy Muscosal Immunology \& Intestinal Microbiota Group. Probiotics and dietary counselling contribute to glucose regulation during and after pregnancy: a randomised controlled trial. Br J Nutr. 2009:101:1679-87.

31. Luoto R, Laitinen $K$, Nermes M, Isolauri E. Impact of maternal probioticsupplemented dietary counselling on pregnancy outcome and prenatal and postnatal growth: a double-blind, placebo-controlled study. Br J Nutr. 2010; 103:1792-9.

32. IImonen J, Isolauri E, Poussa T, Laitinen K. Impact of dietary counselling and probiotic intervention on maternal anthropometric measurements during and after pregnancy: a randomized placebo-controlled trial. Clin Nutr. 2011;30:156-64

33. Luoto $R$, Kalliomäki M, Laitinen $K$, Isolauri $E$. The impact of perinatal probiotic intervention on the development of overweight and obesity: follow-up study from birth to 10 years. Int J Obes. 2010;34:1531-7.

34. Lindsay KL, Kennelly M, Culliton M, Smith T, Maguire OC, Shanahan F, et al. Probiotics in obese pregnancy do not reduce maternal fasting glucose: A double-blind, placebo-controlled, randomized trial (Probiotics in Pregnancy Study). Am J Clin Nutr. 2014;99:1432-9.

35. Hanson L, Vandevusse L, Duster M, Warrack S, Safdar N. Feasibility of oral prenatal probiotics against maternal Group B Streptococcus vaginal and rectal colonization. J Obstet Gynecol Neonatal Nurs. 2014:43:294-304

36. Stojanović N, Plećaš D, Plešinac S. Normal vaginal flora, disorders and application of probiotics in pregnancy. Arch Gynecol Obstet. 2012;286:325-32.

37. Mastromarino P, Vitali B, Mosca L. Bacterial vaginosis: a review on clinical trials with probiotics. New Microbiol. 2013;36:229-38.

38. Moghaddam NM. Recto-vaginal colonization with Group B Streptococcus in pregnant women referred to a hospital in Iran and its effect on Lactobacillus normal flora. J Biol Sci. 2010;10:166-9.

39. Yudin $\mathrm{MH}$, Money DM. Screening and management of bacterial vaginosis in pregnancy. J Obstet Gynaecol Can. 2008;30:702-8.

40. Othman M, Alfirevic Z, Neilson JP. Probiotics for preventing preterm labour. CochraneDatabase Syst. Rev. 2007; Art. No.: CD005941. DOI: 10.1002/ 14651858.CD005941.

41. Carey J, Klebanoff M, Hauth J, Hillier S, Thom E, Ernest J, et al. Metronidazole to prevent preterm delivery in pregnant women with asymptomatic bacterial vaginosis. N Engl J Med. 2000;342:534-40.

42. Anukam K, Osazuwa E, Ahonkhai I, Ngwu M, Osemene G, Bruce AW, et al. Augmentation of antimicrobial metronidazole therapy of bacterial vaginosis with oral probiotic Lactobacillus rhamnosus GR-1 and Lactobacillus reuteri RC-14: randomized controlled trial. Microbes Infect. 2006;8:1450-4. 
43. MacPhee R, Hummelen R, Bisanz J, Miller W, Reid G. Probiotic strategies for the treatment and prevention of bacterial vaginosis. Expert Opin Pharmacother. 2010;11:2985-95.

44. Rodriguez-Granger J, Alvargonzalez JC, Berardi A, Berner R, Kunze M, Hufnagel $M$, et al. Prevention of group B streptococcal neonatal disease revisited. the DEVANI European project. Eur J Clin Microbiol Infect Dis. 2012; 31:2097-104.

45. Javanmanesh F, Eshraghi N. Prevalence of positive recto-vaginal culture for Group B streptococcus in pregnant women at 35-37 weeks of gestation. Med J Islam Repub Iran. 2013;27:7-11.

46. Grimwood K, Stone PR, Gosling IA, Green R, Darlow BA, Lennon DR, et al. Late antenatal carriage of Group B Streptococcus by New Zealand women. Aust NZ J Obstet Gynaecol. 2002;42:182-6.

47. Goldenberg RL, Thompson C. The infectious origins of stillbirth. Am J Obstet Gynecol. 2003;189:861-73.

48. Simonsen KA, Anderson-Berry AL, Delair SF, Dele Davies H. Early-onset neonatal sepsis. Clin Microbiol Rev. 2014:27:21-47.

49. Campbell N, Eddy A, Darlow B, Stone P, Grimwood K. The prevention of early-onset neonatal group $B$ streptococcus infection: technical report fron the New Zeland GBS Consensus Working Party. NZ Med J. 2004;117:1-19.

50. Acikgoz ZC, Gamberzade S, Gocer S, Ceylan P. Inhibitor effect of vaginal lactobacilli on group B streptococcus. Mikrobiyol Bul. 2005;39:17-23.

51. Zárate G, Nader-Macias ME. Influence of probiotic vaginal lactobacilli on in vitro adhesion of urogenital pathogens to vaginal epithelial cells. Lett Appl Microbiol. 2006:43:174-80.

52. Plascencia L, García HS, Lacroix M, Rosa B, Uscanga A. Effect of culture medium on bacteriocin production by Lactobacillus rhamnosus HN001 and Lactobacillus reuteri ATCC 53608. J Microbiol Biotechnol Food Sci. 2013;2:2462-8.

53. Borre YE, O'Keeffe GW, Clarke G, Stanton C, Dinan TG, Cryan JF. Microbiota and neurodevelopmental windows: Implications for brain disorders. Trends Mol Med. 2014;20:509-18.

54. Foster JA, McVey Neufeld K-A. Gut-brain axis: how the microbiome influences anxiety and depression. Trends Neurosci. 2013;36:305-12.

55. Cryan JF, Dinan TG. Mind-altering microorganisms: the impact of the gut microbiota on brain and behaviour. Nat Rev Neurosci. 2012;13:701-12.

56. Marques TM, Cryan JF, Shanahan F, Fitzgerald GF, Ross RP, Dinan TG, et al. Gut microbiota modulation and implications for host health: Dietary strategies to influence the gut-brain axis. Innov Food Sci Emerg Technol. 2014;22:239-47.

57. Bravo JA, Forsythe $P$, Chew MV, Escaravage E, Savignac HM, Dinan TG, et al. Ingestion of Lactobacillus strain regulates emotional behavior and central GABA receptor expression in a mouse via the vagus nerve. Proc Natl Acad Sci. 2011;108:16050-5.

58. Messaoudi M, Violle N, Bisson JF, Desor D, Javelot H, Rougeot C. Beneficial psychological effects of a probiotic formulation (Lactobacillus helveticus R0052 and Bifidobacterium longum R0175) in healthy human volunteers. Gut Microbes. 2011:2:256-61.

59. Benton D, Williams C, Brown A. Impact of consuming a milk drink containing a probiotic on mood and cognition. Eur J Clin Nutr. 2007;61:355-61.

60. Tillisch K, Labus J, Kilpatrick L, Jiang Z, Stains J, Ebrat D, et al. Consumption of fermented milk product with probiotic moduates brain activity. Gastroenterology. 2013;144(7):1394-401.

61. O'hara MW, Swain AM. Rates and risk of postpartum depression-a meta-analysis. Int Rev Psychiatry. 1996;8:37-54.

62. Dennis CL, Doswell T. Psychosocial and psychological interventions for preventing postpartum depression. Cochrane database Syst. Rev. 2013; Art. No.: CD001134. DOI: 10.1002/14651858.CD001134.

63. Dias CC, Figueiredo B. Breastfeeding and depression: A systematic review of the literature. J Affect Disord. 2015;171:142-54.

64. Vliegen N, Casalin S, Luyten P. The course of postpartum depression: a review of longitudinal studies. Harv Rev Psychiatry. 2014;22:1-22.

65. Themeau TM, Grambsch PM. Modeling survival data: extending the Cox Model. New York: Springer; 2000.

66. European Task Force on Atopic Dermatitis. Severity Scoring of Atopic Dermatitis: The SCORAD Index. Dermatology. 1993;186:23-31.

67. Allsworth JE, Peipert JF. Prevalence of bacterial vaginosis: 2001-2004 National Health and Nutrition Examination Survey data. Obstet Gynecol. 2007:109:114-20.

68. Waldie KE, Peterson ER, D'Souza S, Underwood L, Pryor JE, Carr PA, et al. Depression symptoms during pregnancy: Evidence from Growing Up in New Zealand. J Affect Disord. 2015;186:66-73.
69. Williams HC, Burney PGJ, Hay RJ, Archer CB, Shipley MJ, Hunter JJA, et al. The UK Working Party's Diagnostic Criteria for Atopic Dermatitis. Br J Dermatol. 1994;131:383-96.

70. Nugent RP, Krohn MA, Hillier SL. Reliability of diagnosing bacterial vaginosis is improved by a standardized method of gram stain interpretation. J Clin Microbiol. 1991;29:297-301.

71. Cox JL, Holden JM, Sagovsky R. Detection of post natal depression: development of the 10-item Edinburgh Postnatal Depression Scale. Br J Psychiatry. 1987;150:782-6.

72. Marteau TM, Bekker H. The development of a six-item short-form of the state scale of the Spielberger State-Trait Anxiety Inventory (STAI). Br J Clin Psychol. 1992;31(Pt 3):301-6.

73. WHO Expert Committee. Physical Status: the use and interpretation of anthropometry. Technical Report Series No. 854. Geneva: World Health Organisation; 1995.

74. Ramírez-Santana C, Pérez-Cano FJ, Audí C, Castell M, Moretones MG, LópezSabater MC, et al. Effects of cooling and freezing storage on the stability of bioactive factors in human colostrum. J Dairy Sci. 2012;95:2319-25.

75. Mclnnes P, Cutting M. Manual of Procedures for the Human Microbiome Project. Core Microbiome Sampling Protocol A HMP Protocol \#07-001. 2010. http://hmpdacc.org/doc/HMP_MOP_Version12_0_072910.pdf. Accessed 27 July 2015

76. Dekker JW, Wickens K, Black PN, Stanley T, Mitchell EA, Fitzharris P, et al. Safety aspects of probiotic bacterial strains Lactobacillus rhamnosus HN001 and Bifidobacterium animalis subsp. lactis HN019 in human infants aged 0-2 years. Intern Dairy J. 2009;19:149-54.

77. Dugoua J-J, Machado M, Zhu X, Chen X, Koren G, Einarson TR. Probiotic safety in pregnancy: A systematic review and meta-analysis of randomized controlled trials of Lactobacillus, Bifidbacterium, and Saccharomyces spp. J Obstet Gynaecol Can. 2009;31:542-52.

78. Kaplas N, Isolauri E, Lampi A-M, Ojala T, Laitinen K. Dietary counseling and probiotic supplementation during pregnancy modify placental phospholipid fatty acids. Lipids. 2007;42:865-70.

79. Campbell DE. Probiotics and primary prevention of atopic disease: are we closer to a firm evidence base for clinical use? Clin Exp Allergy. 2013; 43:978-80.

80. Fiocchi A, Pawankar R, Cuello-Garcia C, Ahn K, Al-Hammadi S, Agarwal A, et al. World Allergy Organization-McMaster University Guidelines for Allergic Disease Prevention (GLAD-P): Probiotics. World Allergy Organ J. 2015;8:1-13.

\section{Submit your next manuscript to BioMed Central and we will help you at every step:}

- We accept pre-submission inquiries

- Our selector tool helps you to find the most relevant journal

- We provide round the clock customer support

- Convenient online submission

- Thorough peer review

- Inclusion in PubMed and all major indexing services

- Maximum visibility for your research

Submit your manuscript at www.biomedcentral.com/submit 